\title{
RADII OF UNIVALENCE, STARLIKENESS, AND CONVEXITY
}

\section{ShinjI Yamashita}

Let a function $f(z)=z+\sum_{2}^{\infty} a_{n} z^{n}$ be regular in the disk $|z|<1$. The radius of univalence $0.164 \ldots$ of the family of $f$ with $\left|a_{n}\right| \leq n \quad(n \geq 2)$ is, actually, the radius of star-

likeness. The radius of univalence $I-[K /(I+K)]^{\frac{3}{2}}$ of the family of $f$ with $\left|a_{n}\right| \leq K \quad(n \geq 2)$, where $K>0$ is a constant, is, actually, the radius of starlikeness. The radii of convexity of the two families are estimated from below.

\section{Introduction}

Let $N$ be the family of functions $f$ regular in $D=\{|z|<1\}$ with the Taylor expansion

$$
\hat{J}(z)=z+\sum_{n=2}^{\infty} a_{n} z^{n}
$$

Let $F$ be a non-empty subfamily of $N$. The largest number $u(F)$ of $r$, $0<r \leq 1$, such that each $f \in E$ is univalent in $D(r)=\{|z|<r\}$, is called the radius of univalence of $F$. The radius of starlikeness $s(F)$ and that of convexity $c(F)$ of $F$ are defined on adding further the condition that the image $f(D(r))$ is star-shaped with respect to the origin, and the condition that $f(D(x))$ is convex, respectively.

Received 27 January 1982. 
Evidently, $e(F) \leq s(F) \leq u(F)$. The determination of $u(F), s(F)$ and $c(F)$ has been one of the subjects in the theory of univalent functions; see, for example, [1]. It is well-known that $s(S)=\tanh (\pi / 4)$ and $c(S)=2-\sqrt{3}$ for the family $S$ of all univalent members of $N$.

Let $B$ be the family of $f$ of (1.1) with $\left|a_{n}\right| \leq n$ for all $n \geq 2$. Let $K>0$ be a constant and let $G(K)$ be the family of $f$ of (1.1) with $\left|a_{n}\right| \leq K$ for all $n \geq 2$. Gavrilov [3, Theorems 1 and 1'] proved that $u(B)=r_{0}$, where $r_{0}$ is the root in the interval $(0,1)$ of the equation $2(1-r)^{3}-(1+r)=0$, and that $u(G(K))=r_{1} \equiv I-[K /(1+K)]^{\frac{2}{2}}$. Gavrilov's estimate $0.125<r_{0}<0.130$ is erroneous because $r_{0}=0.164 \ldots$. We first improve his results.

THEOREM 1. The identities $u(B)=s(B)$ and $u(G(K))=s(G(K))$ hold.

Next we investigate the lower bounds of $c(B)$ and $c(G(K))$.

THEOREM 2. Let $r_{2}=0.090 \ldots$ be the root in $(0,1)$ of the equation $2(1-r)^{4}-\left(1+4 r+r^{2}\right)=0$, and let $r_{3}$ be the root in $(0,1)$ of the equation $\left(1+K^{-1}\right)(1-r)^{3}-(1+r)=0$. Then $c(B) \geq r_{2}$ and $c(G(K)) \geq r_{3}$.

We note that $(2-\sqrt{3}) r_{0}=0.04 \ldots<r_{2}$ and $(2-\sqrt{3}) r_{1}<r_{3}$. The latter inequality needs a proof.

\section{Proofs}

We shall make use of the following lemma due to Alexander and Remak; see [4, Theorem 1] and [2, Theorem 3].

LEMMA AR. If $h(z)=z+\sum_{n=2}^{\infty} b_{n} z^{n}$ is a member of $N$ and if

$$
\sum_{n=2}^{\infty} n\left|b_{n}\right| \leq 1
$$

then $h$ is univalent and starlike in $D$, while $i_{\hat{J}}$ 


$$
\sum_{n=2}^{\infty} n^{2}\left|b_{n}\right| \leq 1
$$

then $h$ is univalent and convex in $D$.

Proof of Theorem 1. Since $s(B) \leq u(B)=r_{0}$, it suffices to observe that $r_{0} \leq s(B)$. For this purpose let $0<r \leq r_{0}$, and let $f$ of (1.I) be a member of $B$. On applying Lemma AR to $h(z)=r^{-1} f(r z)$, together with

$$
\sum_{n=2}^{\infty} n\left|a_{n}\right| r^{n-1} \leq \sum_{n=2}^{\infty} n^{2} r^{n-1} \leq \sum_{n=2}^{\infty} n^{2} r_{0}^{n-1}=\left(1+r_{0}\right) /\left(1-r_{0}\right)^{3}-1=1,
$$

one can conclude that $h$ is univalent and starlike in $D$, or, $f$ is starlike in the disk $D(r)$. Therefore $r_{0} \leq s(B)$.

For the proof of $s(G(K))=u(G(K))=r_{1}$ we note that $s(G(K)) \leq r_{1}$. For the proof of the converse we let $f$ of (1.1) be a member of $G(K)$ and let $0<r \leq r_{1}$. On applying Lemma AR to $h(z)=r^{-1} f(r z)$, together with

$$
\sum_{n=2}^{\infty} n\left|a_{n}\right| r^{n-1} \leq K \sum_{n=2}^{\infty} n r_{1}^{n-1}=K\left[\left(1-r_{1}\right)^{-2}-1\right]=1
$$

one observes that $h$ is univalent and starlike in $D$, or, $f$ is starlike in $D(r)$. Therefore $r_{1} \leq s(G(K))$.

Proof of Theorem 2. For $r, 0<r \leq r_{2}$, for $f \in B$ and for $z \in D$, we set $h(z)=p^{-1} f(r z)$. By Lemma AR, together with the estimate

$$
\sum_{n=2}^{\infty} n^{2}\left|a_{n}\right| r^{n-1} \leq \sum_{n=2} n^{3} r_{2}^{n-1}=\left(1+4 r_{2}+r_{2}^{2}\right) /\left(1-r_{2}\right)^{4}-1=1
$$

one observes that $h$ is univalent and convex in $D$, whence the same is true of $f$ in $D(r)$. Therefore $r_{2} \leq c(B)$.

For $r, 0<r \leq r_{3}$, for $\hat{f} \in G(K)$ and for $z \in D$, we set $h(z)=x^{-1} f(r z)$. By Lemma AR, together with the estimate 


$$
\sum_{n=2}^{\infty} n^{2}\left|a_{n}\right| r^{n-1} \leq K \sum_{n=2}^{\infty} n^{2} r_{3}^{n-1}=K\left[\left(1+r_{3}\right) /\left(1-r_{3}\right)^{3}-1\right]=1
$$

one observes that $h$ is univalent and convex in $D$, whence the same is true of $f$ in $D(r)$. Therefore $r_{3} \leq c(G(K))$.

REMARK. For $f$ of (I.I) we set

$$
f_{n}(z)=z+\sum_{k=2}^{n} a_{k} z^{k} \quad(n \geq 2) .
$$

If $f \in B$, then the partial sum $f_{n} \in B$ for all $n \geq 2$. Therefore Gavrilov's assertion on $f_{n}$ in [3, Theorem 1] is superfluous. The same is true of $f_{n}$ for $f \in G(K)$ in [3, Theorem 1'].

It remains to prove that

$$
(2-\sqrt{3})(1-\alpha)=(2-\sqrt{3}) r_{1}<r_{3},
$$

where $\alpha=[K /(1+K)]^{\frac{3}{2}}$. Since the function $\varphi(x)=(1-x)^{3 /(1+x)}$ is decreasing for $0 \leq x \leq 1$, and since $\varphi\left(r_{3}\right)=\alpha^{2}$, it suffices to observe that

$$
\varphi((2-\sqrt{3})(1-\alpha))>\alpha^{2} \text {, or } \Phi(\alpha)>0 \text {, }
$$

where

$$
\Phi(x)=(14-8 \sqrt{3}) x^{3}+(-30+17 \sqrt{3}) x^{2}+(21-12 \sqrt{3}) x+(-5+3 \sqrt{3})
$$

for $0 \leq x \leq 1$. As is easily checked, $\Phi^{\prime}(x)=0$ has only one solution $\lambda$ in $0<x<1$, and $\Phi$ is increasing (decreasing, respectively) in $[0, \lambda]([\lambda, 1]$, respectively). Since $\Phi(0)>0=\Phi(1)$, one can assert that $\Phi(\alpha)>0$. 


\section{References}

[1] Ф.Г. Авхаднев, Л.А. Амсентьев, "Основные результаты в достаточных условнях однолистностн аналитичесних фуннций", Uspehi Mat. Nauk 30 (1975) no. 4, 3-60. English translation: F.G. Avhadiev, L.A. Aksent'ev, "The main results on sufficient conditions for an analytic function to be schlicht", Russian Math. Surveys 30 (1975), no. 4, 1-63.

[2] J. Clunie and F.R. Keogh, "On starlike and convex schlicht functions", J. Lordon Math. Soc. 35 (1960), 229-233.

[3] В.Н. Гаврилов [V.I. Gavrilov], "Замечания о радиусе однолистностн голоморфных фуннций" [Remarks on the radius of univalence of holomorphic functions", Mat. Zametki 7 (1970), 295-298.

[4] A.W. Goodman, "Univalent functions and nonanalytic curves", Proc. Amer. Math. Soc. 8 (1957), 598-601.

Department of Mathematics,

Tokyo Metropolitan University,

Fukazawa,

Setagaya,

Tokyo 158,

Japan. 